\title{
DESIGN AND OPERATION OF THE ELECTRON BEAM ION TRAP
}

UCRL-ID--104990

\author{
David Vogel*
}

DE91 000074

High Temperature Physics Division

University of California, Lawrence Livermore National Laboratory, Livermore, CA 94550

Abstract

This report describes the basic features and operating principles of the Electron Beam lon Trap. The differences between EBIT and other sources of highly charged ions are outlined. Its features and operating parameters are discussed. The report also explains why certain design choices were necessary and the constraints involved in building an electron beam ion trap. EBIT's evaporation cooling system is described in detail.

* The author, an Air Force military research associate, alas been working with the EBIT group since 1989. This document was written in June 199() as an introductory description for those interested in details of EBIT's operation. 


\section{Introduction}

The Electron Beam Ion Trap (EBIT) is a unique instrument that is enabling unprecedented experiments with highly charged ions. EBIT consists of an electron beam that strips electrons from low-charge ions. The space charge of the beam confines the ions, and collisions with beam electrons excite $x$-ray transitions in the trapped ions. By measuring the wavelengths and intensities of emitted $\mathrm{x}$-rays, we have been able to determine transition energies and relative excitation cross-sections for ions with charges of +20 and higher. EBIT experiments have also provided cross-sections for dielectronic recombination, and recently we have begun to examine impact ionization processes. Highly-charged ions could also be observed using highenergy accelerators or high temperature plasmas, but EBIT offers advantages over both.

Accelerators such as the Berkeley HILAC and the UNILAC in West Germany manufacture high-charge ions by directing an energetic beam of low-charge ions toward a thick carbon foil; collisions strip additional electrons from the ions. Collisions in a second, thinner, foil produce ions in excited levels, whose decays result in observable x-rays. Generating ions with EBIT requires far less input energy than an accelerator. Achieving a given interaction energy (that is center-of-mass energy) takes less energy when the particles accelerated are electrons rather than heavy nuclei. ${ }^{1}$ Whereas energies of several GeV are needed to accelerate heavy ions to a momentum high enough that electron-ion collisions result in further ionization, the EBIT electrons need only as much energy as the ionization energy of the last electron removed. Thus, although EBIT's maximum accelerating voltage is about $28 \mathrm{keV}$, we have been able to generate and observe neon-like ionization states of barium $\left(\mathrm{Ba}^{46+}\right)$, platinum $\left(\mathrm{Pt}^{68+}\right)$, gold $\left(\mathrm{Au}^{69+}\right)$, and even uranium ( $\left.\mathrm{U}^{82+}\right)$. Holding the ions (essentially) stationary also simplifies analysis of the $\mathrm{X}$-ray spectra. X-ray lines are easier to measure than those from beam-foil experiments because their wavelengths are not Doppler shifted. Beam-foil experiments do provide a higher $X$-ray flux, but in EBIT ions repeatedly undergo excitation and re-emission, while an ion beam produces $x$-rays only in bursts, so that the ions are used only once. On the other hand, accelerated ion beams can still reach higher charge states (e.g. hydrogen-like uranium), and they also allow spectroscopy of the interaction products.

Most existing data on high-charge ions have been obtained using plasma sources. Many sources of high temperature plasmas exist: tokamaks; theta pinches, vacuum sparks, etc. It is the need to understand atomic processes in these devices that motivates much of the research on highly charged ions. As a device for observing ions, EBIT enjoys several advantages over plasmas. One major difference is that EBIT can establish a narrower charge balance; the majority of the ions are in the same ionization state. Additionally, under good conditions, we have only one element at a time in the trap. Perhaps even more important, the narrowness of EBIT's electron energy distribution permits us to isolate specific interactions, while in a plasma the energy distribution is essentially thermal, so that a large number of processes take place at once. And finally, the ion temperature is lower in EBIT. That means Doppler broadening is insignificant, so we can better resolve the $x$-ray lines of interest.

1 The interaction energy $E$ of a two-particle system is the total kinetic energy in the center-of-mass Irame, which equals $E_{0}\left(1-m_{1} /\left(m_{1}+m_{2}\right)\right)$, where $m_{1}$ is the energy of the moving particle and $E_{0}$ its encrgy in the laboralory frame. If $\mathrm{m}_{1}$ " $\mathrm{m}_{2}$, as in EBIT, where $\mathrm{m}_{1}$ is the electron's mass, E $\approx \mathrm{E}_{\text {) }}$. For a heavy ion beam, however, m1"m2, and $\mathrm{L}$ is only a fraction of Eo. 


\section{Development History}

Low energy electron beams have been used as sources of high-charge ions in several laboratories, but E. D. Donets in the Soviet Union was the first to show the value of an electron beam ion source (EBIS) for atomic measurements [5]. Electron beams generate highly charged ions by successive electron impacts. After ionization, the high-charge ions are extracted in order to study their interaction with electrons or other ions, or for injection into accelerators. EBIT is the only such device designed to trap ions indefinitely for $\mathrm{x}$-ray spectroscopy, hence the name Electron Beam Ion Trap. The confined ions are monitored by means of the $x$-rays they emit. The long trapping time permits EBIT to reach higher charge states than conventional electron beam. sources. Moreover, EBIT generates higher ion densities for all charge states.

EBIT was itself an outgrowth of an attempt to study high-charge ions in an electron beam ion source at Lawrence Berkeley Laboratory. When the Berkeley EBIS failed to produce ions with a high enough charge state, Levine, Marrs, and Schmieder traced the problem to instabilities in the electron beam [8], which heated the ions so rapidly that they diffused out of the beam before becoming sufficiently ionized. An examination of beam heating by Litwin, Vella, and Sessler [9] showed that one type of instability, the modified rotational two-stream (RTS) instability, could only be minimized by reducing the beam length. The RTS instability is an inherent characteristic of the trap itself and can only be overcome by making the trap shorter than the instability growth distance. Accordingly, Levine and Marrs designed EBIT to have an axial trap length of only two centimeters. ${ }^{2}$ Reducing the length was a particularly auspicious solution; besides eliminating the RTS heating problem, it also minimized other beam instabilities.

The fundamental differences between EBIT and electron beam ion sources are therefore three: (1) "indefinite" trapping of the ions, (2) the shorter trap length, and (3) access for $x$-ray spectroscopy. Since the ions from an EBIS are examined after leaving the electrostatic trap, they can be filtered through an electromagnetic separation system to select a specific charge state. Moreover, transporting the ions makes electron or ion spectroscopy possible. ${ }^{3}$ Nevertheless, EBIT's ability to produce large numbers of ions with higher charges gi 'es it a significant advantage over the EBIS. The EBIT setup is ideal for $\mathrm{x}$-ray spectroscopy of highly charged ions and for observing processes such as electron impact excitation or dielectronic recombination. Even ionization and recombination measurements may be easier in EBIT than in other apparatus.

2 The plasma oscillation wavelength is equal to $2 v_{\mathrm{e}} / \sqrt{ } 3\left(\omega_{\mathrm{pe}} \omega_{\mathrm{pl}}{ }^{2} / 2\right)^{1 / 3}$, where $\omega_{\mathrm{pe}}$ and $\omega_{\mathrm{pi}}$ are the electron and ion plasma frequencies and $v_{e}$ the electron velocity $|8|$. wpe and $w_{p i}$ depend in turn on the electron and ion densities, and $w_{p}$ on the ion charge and mass [2]. Under normal operating conditions, the oscillation wavelength in EBIT a anges from 1 t $6 \mathrm{~cm}$.

3 All measurements from EBIT so far have been at $x$-ray wavelengths; it is not currently configured to olserve visible or ult raviolet radiation. Most of olir measurements use either semiconductor detectors or crystal spectrometers. 


\section{EBIT Design and Construction}

The major components of EBIT are shown in Figure 1. The electron beam travels upward from the electron gun, through the drift tubes, and into the collector. Metal ions are generated in a metal vapor vacuum arc (MEVVA) and guided into the trap region between the drift tubes. The electron gun, drift tubes, and collector all lie on the axis of a magnetic field established by superconducting Helmholtz coils. The magnetic field is needed to compress the beam and increase its current density. A bias on the drift tubes accelerates electrons to their full interaction energy; it is in the drift tube region that ions are "trapped." The total beam length is about $50 \mathrm{~cm}$, and the entire apparatus is 1.5 meter long.

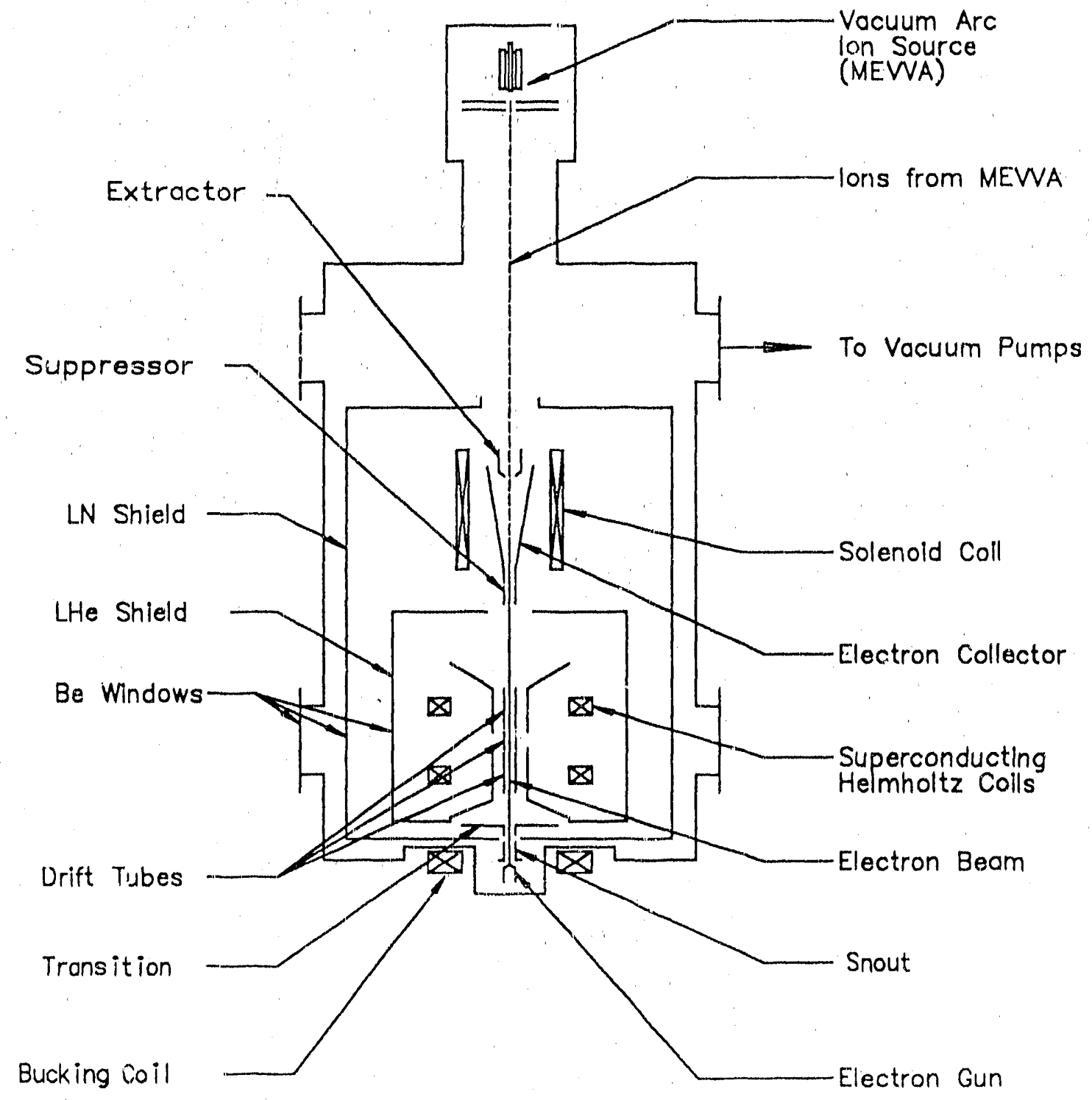

Fig. 1. Basic components of the Electron Beam Ion Trap 


\section{Electron Gun}

EBIT's electron beam (see Figure 2) originates from a Pierce-type electron gun made by the Raytheon Manufacturing Company (the gun was originally designed for use in microwave tubes). The gun's cathode is a heated tungsten clement, impregnated with barium to reduce the work function. ${ }^{4}$ The current is adjusted by changing the anode voltage. Operated at a temperature of about $1000^{\circ} \mathrm{C}$, the cathode has a perveance of $0.5 \mu$ perv, so an anode voltage of $3.4 \mathrm{kV}$ produces about $100 \mathrm{~mA}$, a typical operating current. The cathode's maximum output is

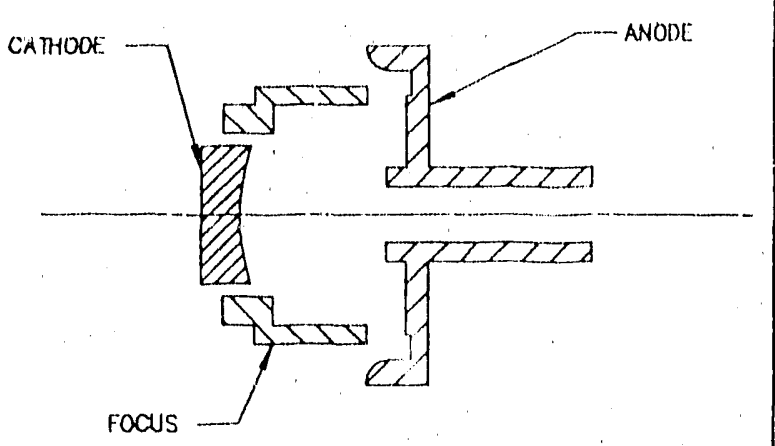

Fig. 2. Electron Gun Geometry about $200 \mathrm{~mA}$, but beam instabilities limit EBIT's operating current to lower values. A Pierce gun ${ }^{5}$ has a spherical cathode so that the emitted electrons converge to a single point, then diverge. Thus, while the cathode diameter is $3 \mathrm{~mm}$, the electrons are focused into a spot of radius $0.36 \mathrm{~mm}$. Since the cathode is only a partial sphere, an additional electrode, the focus electrode, is required to make the potential (nearly) identical to that of a spherical charge distributiun. A separate power supply keeps the focus potential at $-12 \mathrm{~V}$. Surrounding the gun is a magnetic bucking coil, which cancels any magnetic field from the Helmholtz coils, maintaining zero field at the cathode, a necessary condition for maximum magrietic compression of the beam.

\section{Magnets}

As the beam enters drift-tube region, the 3-Tesla axial magnetic field compresses it to a diameter of $60-$ microns and a current density of about $4 \mathrm{kA} / \mathrm{cm}^{2}$. The axial field magnitude inside EBIT varies by less than 0.02 percent over the $2-\mathrm{cm}$ length of the ion trap. The niobium-titanium coils must be continually cooled by liquid helium; the helium reservoir and magnet are shielded in turn by a liquid nitrogen barrier. At 3 Tesla, the coils require $150 \mathrm{~A}$, generated by two HewlettPackard power supplies. The field contains approximately $5 \mathrm{~kJ}$ of energy. The power supply outputs are governed by a current controller that ramps the current up over a period of 1-2 minutes, in order to prevent high-voltage sparks. Two pairs of steering coils, mounted on the exterior of the vacuum chamber permit fine adjustments to the magnetic field direction. Each pair's current can be controlled separately to produce a field of several Gauss perpendicular to the electron beam.

Alignment of the magnetic field with respect to the electron beam axis is important in producing a beam without instabilities. The electron gun is mounted rigidly at the bottom of EBIT, while the coils and drift tubes pivot from a ball joint above. After the entire assembly has

4 One drawback to the barium cathode is that the barium eventually becomes depleted. Cathode lifetime with daily usage secms to be several months. The gun also must be kept under vacuum or in an environment of pure nitrogen since oxygen poisons the cathode.

5 Pierce guns are naned after J. R. Pierce who formulated many of the principles used in designing electron beams. His 1954 book on electron beams is still widely used [11]. 
been installed, stainless-steel wires attached to the lower end can be adjusted in order to align the magnetic axis with the gun axis. In the critical region between the gun and trap, the field direction and the mechanical axis are parallel to better than 0.003 radian.

Recently we have driven the Heimholtz coils at higher currents to compress the beam further. The higher field also allows EBIT to run at a higher total beam current. The largest field we achieved (before quenching) was about $3.5 \mathrm{~T}$, at a magnet current of $190 \mathrm{~A}$. The $\mathrm{x}$-ray count rate increases dramatically with beam current (approximately proportional to the current squared). Quenching the magnet is a risky procedure since dumping so much energy at once could potentially damage or distort the Helmholtz coils. Fortunately this has not occurred. Instead the magnet is able to dump all the excess field energy into the liquid helium, boiling it off immediately.

\section{Beam Radius}

The intense magnetic field compresses the electron beam into an extremely small radius. Preliminary calculations predicted that EBIT's electron beam would have a radius of approximately 30 microns; a series of measurements by Levine et al. later confirmed this is so [7]. They measured the ion-beam overlap region by using an imaging slit to map the radial extent of $\mathrm{x}$-ray emission. Since the ions have a broader spatial distribution than the electron beam, the emission width approximately equals the electron beam diameter. The slit has a 30-micron aperture and is mounted $2 \mathrm{~cm}$ from the beam. The beam intensity was mapped with a position-sensitive proportional counter located $50 \mathrm{~cm}$ away from the slit. The beam profile is important since it affects the beam-ion overlap, the electron density, and the space-charge potential.

Electrons launched from an ideal cathode, that is with no thermal energy, into an axial magnetic field would form a beam with radius:

$$
r_{B}=\frac{0.015 \cdot I^{t}}{B \cdot E^{t}}
$$

where $I$ is the beam current, $E$ the electron energy (determined by the drift tubes), and $B$ the axial field magnitude. This ideal situation is called Brillouin flow and $r_{B}$ is the Brillouin radius. In practice, the random thermal transverse motion of the electrons leads to larger beam radii. The effect of thermal motion on electron flow is described by Pierce [11]; a more accurate, semiempirical description was developed in later papers by Herrmann and Amboss [1,6]. Their calculations show that the actual beam profile has a Gaussian distribution in the radial dimension, and that the radius of a cylinder that encloses $80 \%$ of the beam is given by:

$$
R=r_{B}\left\{\frac{1}{2}+\frac{1}{2}\left(\frac{8 k T m_{e} r_{c}^{2}}{\mathrm{e}^{2} B^{2} r_{B}^{4}}+\frac{B_{c}^{2} r_{c}^{4}}{B^{2} r_{B}^{4}}\right)^{3 / 2}\right\}^{3 / 2}
$$

where $B$ is the axial magnetic field, $B_{c}$ the magnetic field at the cathode, $r_{C}$ the cathode radius, $T$ the electron temperature at the cathode, and $r_{B}$ the Brillouin radius. $R$ is called the Herrmann radius and depends strongly on the cathode magnetic field and on the electron thermal energy, but, for sufficiently large temperatures, varies only weakly with $r_{B}$, the Brillouin radius. The radiur is a 
minimum for $B_{c}=0$; this is the reason for the bucking coil. ${ }^{6}$ EBIT's cathode emits electrons with a temperature of $0.1 \mathrm{eV}$, which implies a radius of $31 \mu \mathrm{m}$. Figure 3(a) shows the variation in radius if the cathode field varies slightly from zern, while $3(b)$ shows how a change in $r_{B}$ affects the radius (for $B_{c}=0$ ). Since $r_{B}$ is the only factor influenced by the beam current or accelerating voltage, EBIT's beam profile stays roughly the same over a wide range of current and energy settings.

\section{Space Charge}

Knowing the beam radius accurately is important since the electron space charge establishes the radial electric field that confines the ions within the beam. The smaller the beam radius, the smaller the confinement volume and the larger the electron-ion interaction rate. The potential difference between the axis and the edge of the beam can be calculated using Gauss's Law; the result is:

$$
\mathrm{V}_{\mathrm{r}}=0.55 \cdot \mathrm{I} / \mathrm{V}^{\mathrm{t}} \text { (volts) }
$$

(where I is the beam current in milliamperes, and $\mathrm{V}$ is the drift tube potential in kilovolts); ions with kinetic energy lower than $q_{i} V_{r}$ will remain within the beam ( $q_{i}$ is the ion charge). The current and acceleration voltage thus determine the degree of beam-ion overlap. Similarly, the radial field makes the axial potential lower than the drift tube potential by the amount:

$$
V_{t}=V_{r}\left(1+\ln \left(R / r_{B}\right) \approx 9 \cdot V_{r}\right.
$$

( $R$ is the drift tube radius), which means the interaction energy is slightly different than the accelerating potential. At $100 \mathrm{~mA}$ and a drift tube voltage of $10 \mathrm{kV}, \mathrm{V}_{\mathrm{r}}$ is about $17 \mathrm{~V}$ and $\mathrm{V}_{\mathrm{t}}$ is $140 \mathrm{~V}$. The space charge is reduced by the ions and slightly increased by secondary electrons, so we cannot determine these values exactly from the beam current and voltage (since we cannot measure the ion density), but the interaction energy can be determined by measuring the energy of $x$-rays emitted through radiative capture of beam electrons. The space charge also introduces a spread in our electron-ion interaction energy, since electrons on the beam axis will have less kinetic energy than those at the edge.

6 We have noted recenlly, however, that making the cathode fick nonzero may nasrow the byam's energy width, even though the radius becomes larger. Thus, energy "esolution improves at the cost of lower $x$-ray count ralcs. a)

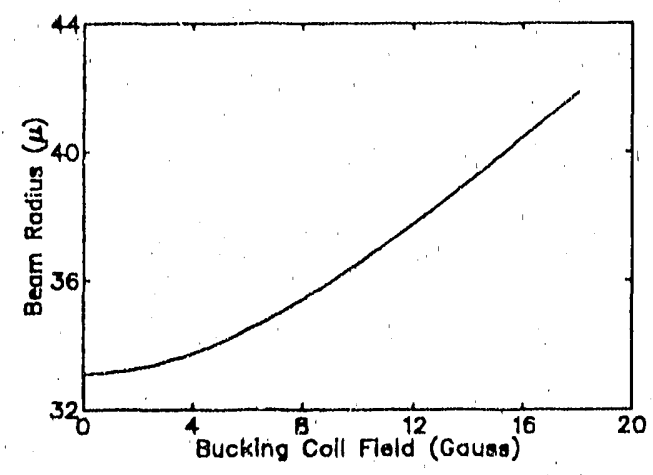

b)

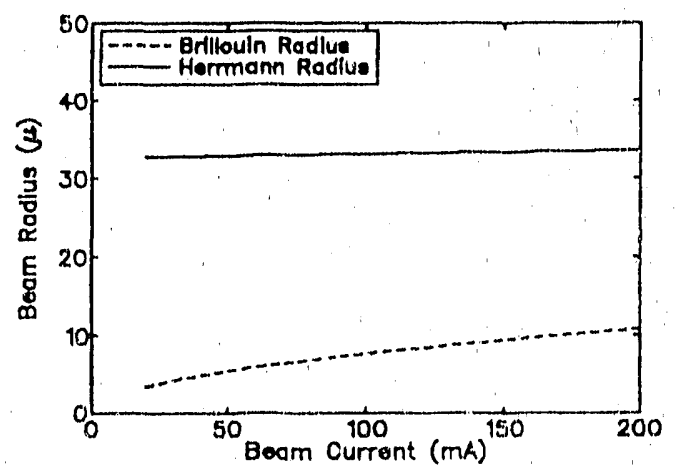

Fig. 3. (a) Electron beam radius as a function of cathode field, $\mathrm{r}_{B}=7.7$ micron $\left(\mathrm{I}_{B}=100 \mathrm{~mA}\right.$, $\mathrm{E}_{\mathrm{i}}=18 \mathrm{keV}$ ). (b) Beam radius as a function of beam current, $E_{i}=18 \mathrm{keV}$. The Herrmann radius is insensitive to the total current. 


\section{High-Voltage Drift Tubes}

The drift tube assembly performs two basic functions: (1) it accelerates the beam electrons to their full interaction energy and (2) it provides an axial electrostatic trap for the positive ions. The assembly comprises three copper tubes, arranged as shown in Figure 4(a). The middle drift tube, DT 2, is $2 \mathrm{~cm}$ long, with an inner diameter of $1 \mathrm{~cm}$. It actually consists of four separate quarter sections; the spaces between them form the $\mathrm{x}$-ray ports. The end drift tubes, DT 1 and 3 , are each $9 \mathrm{~cm}$ long and taper from a $0.3 \mathrm{~cm}$ inner diameter to $1.4 \mathrm{~cm}$ at the outer ends. Each has a counterbore that matches the diameter of the center drift tube. All three are held in place by precision-machined sapphire insulators attached to the helium reservoir.

The beam accelerating voltage is provided by a precision high voltage amplifier that elevates all three drift tubes simultaneously to a potential of up to $30 \mathrm{kV}$ ? $^{7}$ The $\mathrm{HV}$ amplifier combines a $50-\mathrm{kV}$ power supply with a fast (slew rate $=10 \mathrm{kV} / \mathrm{ms}$ ) high-voltage regulator. A floating low-voltage supply superimposes a positive bias (typically $400 \mathrm{~V}$ ) on the two outer drift tubes. This DT 1-3 potential establishes the axial ion trap. The upper drift tube's bias can be further controlled by a second floating power supply, in order to inject ions or dump them from the trap. Both low-voltage amplifiers are controlled through fiber-optic links.

The non-uniform cross-section of the three drift tubes provides an optimal axial well potential. Figure 4(b) shows the approximate profile of EBIT's potential well, while Figure $4(\mathrm{c})$ is the potential that would arise if all three drift tubes had the same constant cross-section. The potential in (b) is better because it confines the ions within a shorter length, thereby achieving a better ion density. Even more important, is the flat bottom it establishes so that all ions in the visible segment of the trap (that is, ions that have a direct line-of-sight through the $\mathrm{x}$-ray ports) have the same energy. An additional benefit of the tapered geometry is that low-charge ions escape more readily, enhancing the evaporation cooling (explained below). The tapered outer drift tubes eliminate the "hump" at each end, thus creating a well with steeper, constantly-sloping sides. The narrower diameter of the outer electrodes necessitates the counterbore cavities, in order to prevent beam electrons that graze the edges from emitting bremsstrahlung $x$-rays through the (a)

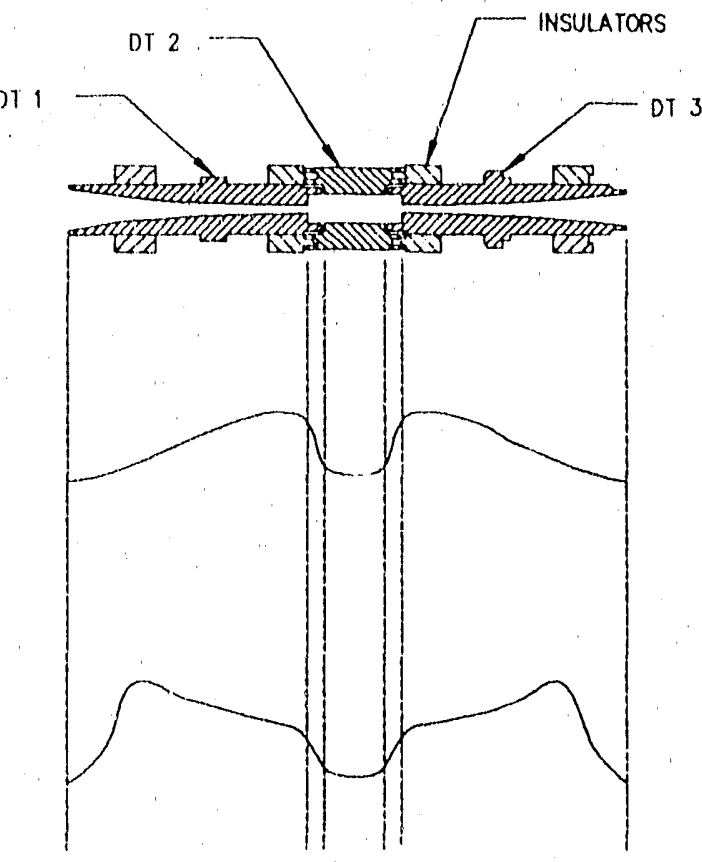

Fig. 4. (a) Drift tubes and supports and (b) the axial potential well established by tapered drift tube geometry, and (c) axial potential that would be produced if the drift tubes had a uniform cross section.

7 In practice, however, low-encrgy penning electrons usually cause high vollage breakdown at $22-25 \mathrm{kV}$. 
windows. The cavities ensure that any bremsstrahlung created occurs well beyond the ports. Making the counterbore depth $0.5 \mathrm{~cm}$ allowed the trap potential to increase smoothly rather than rise in a double step.

The transition and snout electrodes, located above the electron gun, provide a smooth and uniform potential gradient between the gun and drift tubes. They ensure that no electrons are reflected back toward the cathode and that no secondary trap is established between e-gun and drift tubes. In effect, the transition and snout keep electrons from "seeing" ground potential once they leave the cathode. To operate properly, their potentials should be between those of the anode and drift tubes, i.e.:

$$
V_{\text {drtft }}>V_{\text {snout }} \geq V_{\text {traris }} \geq V_{\text {anode }}
$$

Note that this also implies an anode voltage less than the drift tube voltage. That becomes a problem if we want to operate at energies below the desired anode voltage since we then have to settle for lower beam current. When the beam is poorly focused, the snout current, normally only 1-3 $\mu \mathrm{A}$, increases, resulting in potentially unstable operation. The increased snout current probably stems $\mathrm{fr}^{\prime} / \mathrm{m}$ secondary electrons created at the entrance to the collector. We optimize the electron beam by adjusting the steering coils, bucking coil, and (if necessary) the snout and. transition voltages, until the snout current is a minimum. A $10-\mathrm{kV}$ power supply coupled with a four-channel regulator permits dynamic adjustment of the snout and transition voltages. This quad regulator also supplies the high-voltage bias for the MEVVA.

\section{Collector}

After leaving the drift-tube region, beam electrons are eventually dumped on the walls of the collector, a conical cylinder. A separate collector magnet cancels the magnetic field in the collector region making the beam diverge. The collector potential is a constant 1500 volts, which means that the electrons decelerate between the drift tubes and collector, a scheme that reduces the beam power consumption. The collector nevertheless dissipates the residual beam power, about 200-300 watts. Both the collector and its magnet are cooled by liquid nitrogen, provided through a separate feed from the one for the $\mathrm{LN}_{2}$ shield around the superconducting magnet. The suppressor prevents secondary electrons, formed when the beam enters the collector, from traveling back to the drift tubes. Its potential is typically $1000 \mathrm{~V}$. The extractor keeps ions from the MEVVA moving smoothly through the collector and on toward the drift tubes. The extractor potential is -50$) \mathrm{V}$.

\section{Ion Injection}

The metal vapor vacuum arc (MEVVA), located $75 \mathrm{~cm}$ above the collector, is EBIT's primary source of ions. It produres a $20-\mu$ s pulse of metal ions that are guided into the trap region. It can produce low-charge ions of victually any metal. Besides using the MEVYA for metal ions, we can also introduce gases into EBIT. They are injected into the trap ballistically from a gas jet through a differential pumping system, a scheme designed to minimize the load on EBIT's vacuum system. In addition, because of the barium dispenser cathode, the electron beam is a copious source of barium ions. Tungsten is also present to a lesser extent. Both tungsten and barium act as contaminants in experiments with lighter ions, but their density can be reduced by 
frequently dumping and re-injecting a fresh supply of ions. Other contaminants, which appear sporadically, include mercury and argon.

Figure 5 is a cross-sertional view of the MEVVA, which was invented by Brown et al. at Lawrence Berkeley Laboratory $[3,4]$. The anode and focus electrodes are stainless steel, while the cathode, and sometimes the trigger electrode as well, are made of the metal to be ionized. Alumina is used for the insulators. At present, only one metal can be used at a time, but several possibilities exist for generating two or more types of ion, e.g., using alloys or a crystalline compound for the cathode material. ${ }^{8}$ The MEVVA power supply biases the anode, cathode, and trigger to the extraction voltage of $7-8 \mathrm{kV}$. A potential of $150-300 \mathrm{~V}$, the arc voltage, is superimposed between the cathode and anode. A pulse of several kilovolts applied to the trigger electrode by a pulse transformer

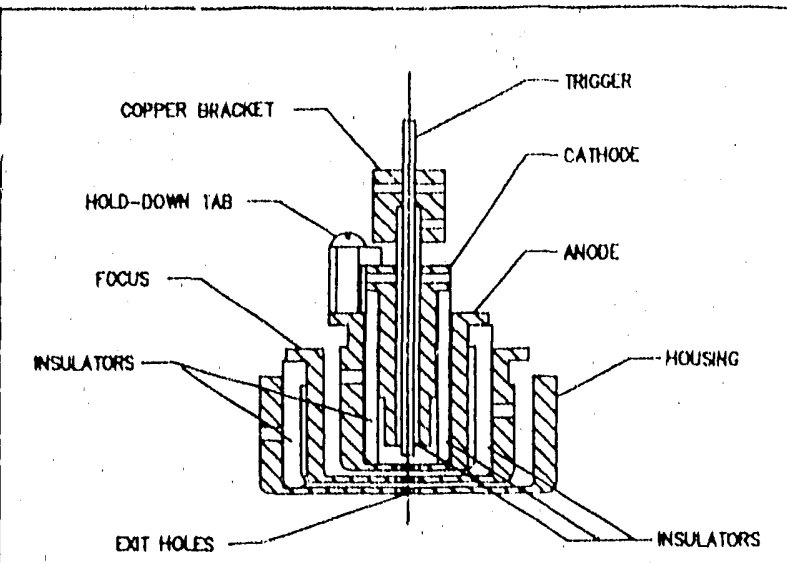

Fig. 5 Cross-sectional view of the MEVVA. Anode, focus, and housing are stainless stecl, while cathode and trigger consist of the metal to be ionized. Insulators are ceramic and hold-down tab is macor. initiates a discharge between cathode and anode.

The MEVVA's operation depends on the restriction of the arc into narrow streamers of current, a characteristir, feature of a vacuum discharge. The current streamers form hot spots on the cathode, which vaporize and expel metal ions into the cathode-anode gap. The discharge itself is composed primarily of electrons, whose space charge is strong enough to pull the positive ions in a direction opposite to the arc voltage (which drops to only 20-100 V during the discharge). As positive ions approach the anode, however, they see the $8 \mathrm{kV}$ extraction voltage and are swept through holes in the anode, creating a beam of ions. The EBIT MEVVA needs about $7 \mathrm{kV}$ to extract ions, but a voltage much higher than $8 \mathrm{kV}$ results in breakdown.

The extracted ion current is approximately $10 \mathrm{~mA}$, which means about a total charge of $2 \times 10^{-7}$ coulomb per pulse. The charge state varies somewhat with the clement, but even for uranium, few ions have a charge as high as +10 . Assuning an average charge of +5 , we would have $2.5 \times 10^{11}$ ions per pulse. Only a fraction make it into the trap, however. Guiding as many ions as possible there is the goal of the MEVVA's focus electrode and the ion extractor (descrioed above) located in the collector. Nevertheless, most of the ions miss because they start out at an oblique angle or from an off-center position, or because they have sufficient energy to continue on past the trap. Moreover, the ion swarm expands to about $6 \mathrm{~cm}$ before reaching the extractor, whose aperture is only $3 \mathrm{~mm}$ wide. Once they reach the collector, the ions are guided and compressed by the electron beam. During ion injection, the drift tubes are biased to the same voltage as the MEVVA, so that the ions will reach the trap with approximately zero velocity. As mentioned above, an additional floating power supply biases DT 3 , in order to "lower the gate" temporarily and admit ions into the trap.

8 Brown et al. [3] were able 10 get lanthanum and boron ions simultaneously with a cathode of lanthanum hexahoride. 
Gas ions are somewhat easier to inject since gases can be admitted into the containment vessel through the evaporation cooling system (described below). Because most of the atoms that make up gas molecules have low atomic numbers, we have done relatively little with thern: EBIT's primary focus so far has been $x$-ray transitions above $1 \mathrm{keV}$. Argon, krypton, and xenon are of interest, of course, and will be investigated. We also plan to try gases that contain heavy elements, such as uranium hexafluoride. We routinely inject $\mathrm{N}_{2}$ for cooling, as outlined below, and oxygen has been used to supply calibration lines for 3-3 transitions in gold and platinum.

The large number of barium ions in the electron beam make it the easiest element to trap. We have found that operating the cathode at a slightly higher heater current increases the barium . content. Unfortunately, it also tends to reduce the gun's lifetime since it increases the barium depletion rate. There is a definite decrease in the number of barium ions as the cathode ages. Barium causes problems in experiments with ions of lower atomic number. The higher-charge barium ions drive out the lower-charge ions we want to observe. Therefore, we must periodically "dump" the trap and inject a fresh supply of ions.

\section{Evaporation Cooling}

One feature that makes EBIT unique, and that improves its performance dramatically, is the cooling provided by lowcharge ions. A fundamental problem with all electron beam ion sources is that the electronion collisions not only lead to ionizations and excitations, they also impart kinetic energy to the ions. The collisions rapidly increase the ion temperature, giving many ions enough energy to drift outside the electron beam, where they are no longer available for interaction. With EBIT, however, it was discovered early on that injecting light ions along with heavier ones dramatically increased the number of high- $Z$ ions in the beam. The lower-charge ions were actually removing kinetic energy from the high-charge ions, that is, lowering their temperature. Schneider et al. performed an experimental investigation of

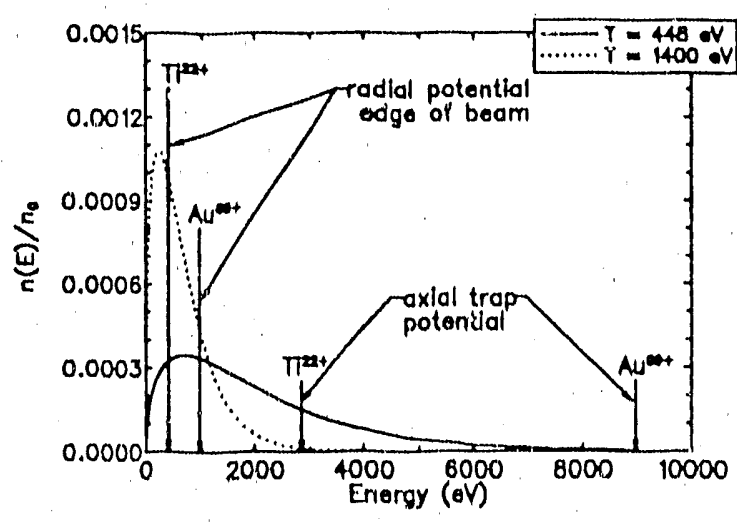

Fig. 6. Evaporation cooling by low-charge ions. Without the low-charge ions, ion temperature is higher, so most of the gold ions orbit outside the beam. Adding the titanium ions reduces the temperature to $448 \mathrm{keV}$, so the higher charge gold ions stay within the beam. this effect, and coined the term "evaporation cooling" for it [12]. Evaporation cooling is now used routinely for almost all EBIT experiments. It allows ions to stay in the electron beam longer and enhances $x$-ray count rates. In the case of heavy ions such as gold or platinum, we also reach a higher charge state than would be possible without cooling.

Figure 6 shows the effect of adding low- $Z$ ions. The dotted-line curve is the ion energy distribution for a mixture of gold and titanium ions. The ions collide frequently enough with each other that kinetic energy is partitioned evenly, so the energy distribution is the same for both types. At a beam energy of $18 \mathrm{keV}$, most of the titanium ions are bare or have only one electron, while a significant fraction of the gold ions reach the neon-like charge state. Vertical lines represent the 
space-charge potentials at the edge of the electron beam for each ion (which are diflerent since they have different charges) and the respective axial potential barriers from the drift tube bias. Since the axial potential barrier is lower for the titanium ions, a significant fraction can escape the well, and their escape rate determines the ion temperature. With cooling, the ion temperature is about $450 \mathrm{eV}$, and most of the gold ions remain in the beam. Without titanium ions, however, the temperature is determined by escaping gold ions and is therefore highar (solid line). In this case, a much lower percentage remains in the beam. In fact, as it turns out, the gold ions do not even stay within the beam long enough to reach the neon-like charge state.

The EBIT cooling system currently uses, not titanium, but nitrogen ions. Nitrogen was initially employed in order to cool lighter ions, such as iron and nickel, but experiments showed it to be more efficient at cooling the heavier ions as well. It was also more convenient to use since gas injection is simpler, and high purity $\mathrm{N}_{2}$ is available at relatively low cost. Computations by Penetrante et al. !ater confirmed that nitrogen is more effective [10]. Subsequent simulations indicate that aluminum or magnesium would remove kinetic energy even more effectively, but $\mathrm{Al}$ and $\mathrm{Mg}$ ions have been difficult to inject so far. ${ }^{9} \mathrm{CO}_{2}$ was tried recently, but the data have not yet been evaluated.

The injection system is designed to provide an adjustable level of cooling while minimizing the gas load on EBIT's vacuum pumps. Before reaching the vacuum chamber, the gas travels through two chambers (see Fig. 7), each equipped with a turbomolecular pump. The pumps lower the injection pressure to about $10^{-7}$ to $10^{-6}$ Torr. An electrically-controlled valve permits adjustment of gas flow into the outer injection chamber and thus controls the injection rate. The ionization gauge pressure gives a relative measurement of the number of ions injected, but unfortunately the system does not allow us to measure how many molecules actually reach the trap. Less than one percent of the gas molecules entering the vacuum vessel help to cool the high-charge ions, however. The appendix explains the cooling system in more detail and derives an estimate for number of cooling ions injected.

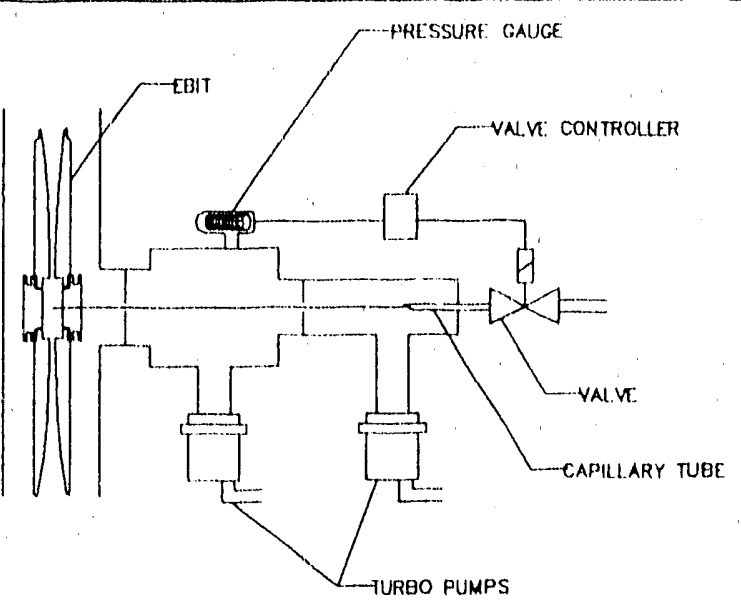

Fig. 7. EBIT gas injection system. The gas injector uses a differential pumpling scheme. Two 50-liter/s turbomolecular pumps reduce the pressure in the inner (left) chamber to 10-7 $-10^{6}$ Torr, minimizing the gas load on EBIT. The inlet valve is a Balzers UDV 135 thermovalve. Controlled by the pressure reading from the ionization gauge, it can be set to maintain a constant pressure in the inner chamber.

The need to have cooling ions escape at an optimum rate: means the axial potential well, determined by the DT $1-3$ voltage, cannot be too deep. Otherwise, the low-charge ions escape too slowly, raising the ion temperature enough that an apprectable? fraction of the high-charge ions follow orbits outside the beam. At first glance it might seem that most ions would escape to the drift tube walls, since the radial potential is

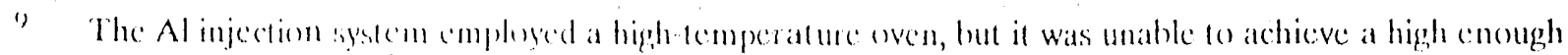

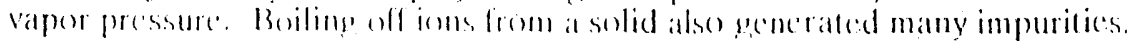


smaller than the axial potential. The radial potential, 10-20 volts at the beam radius, is 90 -180 volts at the drift tube walls, still smaller than the axial barrier of 200-300 volts. But the ions are also constrained radially by the magnetic field, which forces them to travel in eccentric orbits. To reach the wall, they must diffuse both in energy and angular momentum, a slow process [12]. Therefore, unless the axial barrier is very high, most ions leave axially, rather than radially.

\section{Vacuum System}

EBIT"s vacuum system is designed to provide a vacuum of about $10^{-12}$ to $10^{-13}$ Torr inside the drift tubes. Our ionization gauges are good only down to $10^{-10}$ Torr and are located in remote positions, so we cannot verify that the pressure gets this low. Since the low temperature components inside, including the drift tubes themselves, remove a lot of gas, we can be confident that our pressure is well below 1(1-10 Torr. All of EBI'T's internal components employ materials suitable for an ultra high vacuum. The containment vessel, valves, flanges etc. are all stainless steel with copper gasket seals, while the superconducting magnet assembly (the LHe reservoir and the enclosure for the coils) is alumintim. The drift tubes are made of oxygen-free, high-conductivity copper. The system stays con inuously under vacuum except when repairs are needed.

The vacuum vessel has a volume of $3,000-3,500$ cubic inches. Turbomolecular pumps bring it down initially to the $10^{-6}$ 'Torr range, then ion punips take over. The system uses five Varian ion pumps; the two primary pumps have pumping speeds of $45 \mathrm{liter} / \mathrm{s}$ and $30 \mathrm{liter} / \mathrm{s}$. The larger is a Varian Star Cell unit, designed to remove noble gases more effectively. Additionally, $8-1 / \mathrm{s}$ pımps are attached to the electron gun, MEVVA, and residual gas analyzer. ${ }^{10}$ The MEVVA and slectron gun can both be valved off and changed (in about 4-6 hours) without breaking the primary vacuum. EBIT was designed to take advantage of the additional pumping provided by the low-temperature surfaces, and they lower the pressure substantially. ${ }^{11}$ With EBIT warm, we measure a vacuum of about $4 \times 10^{-8}$ Torr, but after cooling with LHe, the gauge pressure drops below its $10^{-10}$ Torr limit. In effect, the $\mathrm{LN}_{2}$ and LHe shield are large cryopumps, and like all cryopumps they need to be reactivated once they have accumulated too much frozen debris. When the system shuts down for brief perion, we allow the liquid helium reservoir to run dry, but the $\mathrm{LN}_{2}$ shield is kept full continuously. Thus, a warm-up is necessary every two months or so, in order to remove material from the ntrogen shield.

\section{Control Program and Data Acquisition}

EBIT uses microcomputers as a front end to both its data acquisition and its electronic control system. The data collection system uses an IBM AT computer, while the control program runs on an IBM XT. The two computers work through a CAMAC interface; the software for both is written in the C progr mming language. Both systems have a graphical user interface.

The control system adjusts the output voltages supplied to the drift tubes and other EBIT subsystems. Within a few milliseconds (response time depends on the power supply), it can change the drift tuhe high voltage, the axial trap potential, the MEVVA extraction voltage, or the anode voltage (and thus the beam current). It also sends trigger pulses to the data acquisition modules,

11" The Hiten quadrupole mass aralyzer identifies trace gases, which helps in troubleshooting vacuum prohlineris

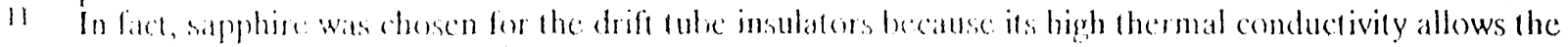
drift rulese for cool monce rapidly. 
in order to synchronize $x$-ray spectra with different segments of the injection cycle. The system can manage up to 16 separate outputs. It controls each voltage level through an analog signal. A summing module is available to combine the outputs from several channels; this allows us to switch the drift tube and DT 1-3 (trap) voltages among several levels. Additionally, each voltage can be switched on or off by the timing signal, a 16-bit digital word that is sent to a restart module. Each bit corresponds to one of the 16 voltages. If its value is one, the restart module keeps the corresponding power supply on; if the bit's val !e is zero, its output is interrupted. The digital signal is generated at a rate set by the operator; 2 milliseconds is a typical clock period.

\section{Clock period $=2.0 \mathrm{~ms} ;$ Total period $=4.5960 \mathrm{sec}$}

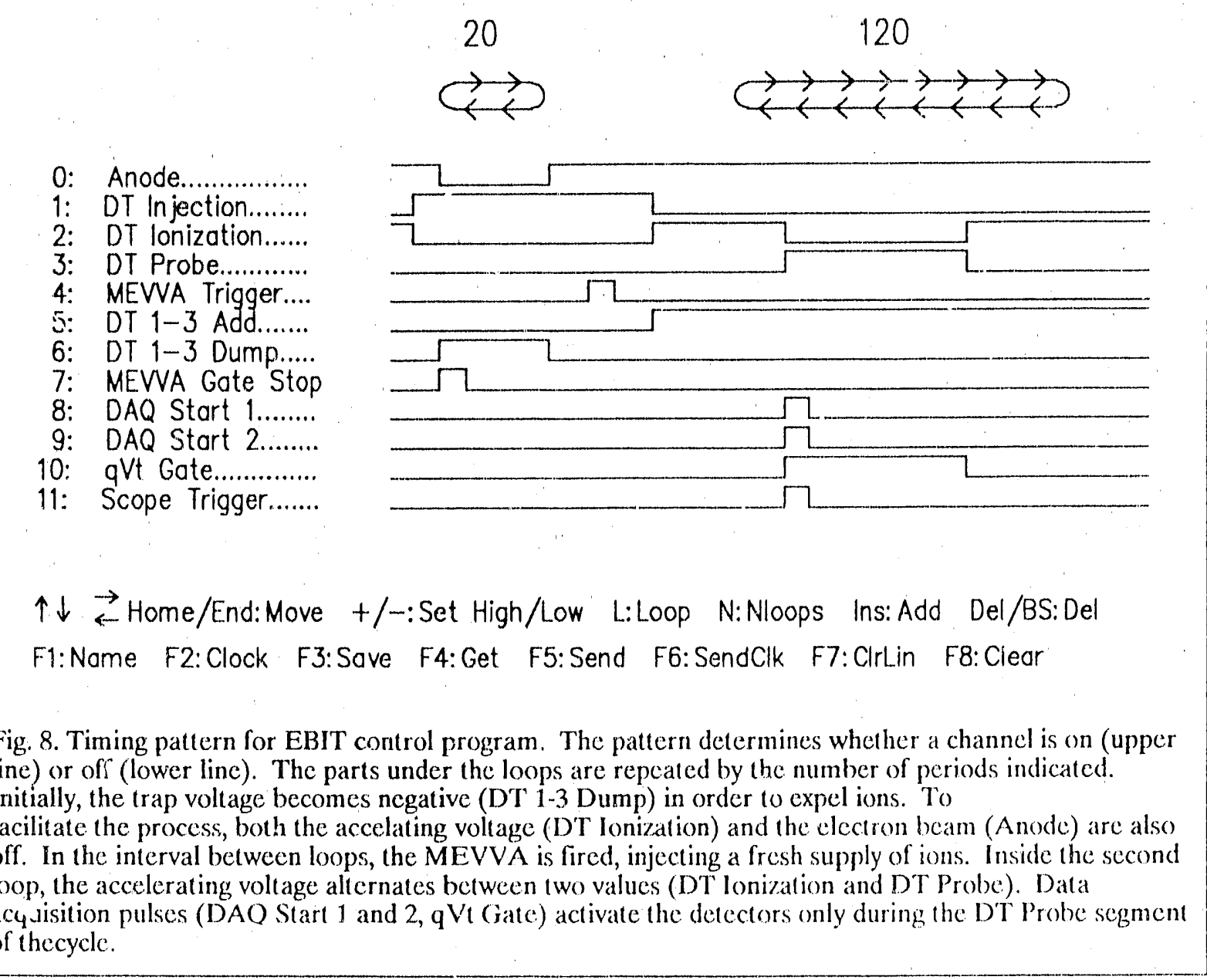

The voltage levels and timing pattern are both controlled through a graphical user interface, which consists of two screen displays. One sets the voltage levels, the other controls the timing pattern. Figure 8 shows an example timing pattern. The user can also specify the length of each time step, which can be set as low as $100 \mu$ s (the HV regulators respond more slowly, however; the drift tube power supply is the slowest, with a slew rate of approximately $10 \mathrm{kV} / \mathrm{ms}$ ). Loops give the user flexibility in specifying a complicated timing patterm. Both the voltage and timing patterns can be saved on disk and recalled. 
EBIT's data açuisition setup can compile data from up to four detectors simultancously, which allows us to use all four $x$-ray ports at once. ${ }^{12}$ The user is free to record, store, and view the results from any data run, including one in progress, without interrupting dat? acquisition. The system incorporates two multichannel analyzers and two analog-to-digital converters (ADC's). Like most experiments, EBIT also uses a combination of coincidence gates, delay generators, single channel analyzers, etc. to reduce the number of background counts. The ADC outputs are fed through a data router into a histogramming memory. "The data router allows the ADC's to divide information from different parts of a timing cycle into separate spectra, up to 32 spectra for each ADC. Our inner-shell ionization measurements rely heavily on this capability, which can show us the effect of changing interaction energy or other parameters. To measure an inner-shell cross-section, we flip the energy between a dielectronic recombination resonance and the ionization level every ten milliseconds. After switching to the higher ionization energy, we record five spectra for two milliseconds each. By comparing the five spectra, we can determine whether ionization changed the charge baiance significantly over the ten-millisecond period.

The EBIT data acquisition program besides controlling the ADC's and multichannel analyzers, also allow's us to perform preliminary analysis on data. It can plot an individual spectrum on the computer screen or sum several spectra. Built-in subroutines allow the user to collapse or smooth a data set, or to zoom in on a portion of the spectrum. One can quickly check the height (number of counts) for any channel, or if energies are known for two or more lines in the spectrum, enter energy calibration coefficients for the channel numbers. Some statistical tools are also available. For instance, the program can compute a background-subtracted sum of the counts between any two channels, calculating a mean and standard deviation for individual peaks. Once a run is complete, the data file is stored on the computer's hard disk in binary form. The binary format produces very compact files; generally a few high-density diskettes suffice for 1-2 weeks of experimental data.

\section{Acknowledgements}

Work performed under the auspices of the U.S. Department of Energy by the Lawrence Livermore National Laboratory under contract No. W-7405-ENG-48. I would lite to thank all the members of the EBIT group for their support and assistance, in particular, Dave Knapp and Peter Beiersdorfer, who contributed many helpful comments and suggestions.

12. In most cases, however, one purt is laten up by the ion conling appatratus. 


\section{REFERENCES}

[1] Kurt Amboss.

Studies of a magnetically compressed electron beam.

IEEE Transactions on Electron Devices, volume ED-16, number 11, pages 897-964, November 1969.

[2] David L. Book.

NRL Plasma Formulary.

Navai Research Laboratory, NRL Publication 0084-4040, page 28, 1987

[3] I. G. Frown, J. E. Galvin, B. F. Gavin, and R. A. MacGill.

Metal $\vee$ anor vacuum arc ion source.

Review of iscientific Instruments, volume 57, number 6, pages 1069-1084, June 1986.

[4] I. G. Briwn, J. E. Galvin, R. A. MacGill, and R. T. Wright.

Miniature high current metal ion source.

Applied Fhysics Letters, volume 49, number 16, pages 1019-1021, 20 October 1986.

[5] E. D. Donets.

Electron beam ion sources and associated physics at JINR.

Nuclear Instrumen.s and Methods in Physics Research, volume B9, pages 522-525, 1985.

[6] Gabriel Herrmarin.

Optical theory of thermal velocity effects in cylindrical electron beams.

Joumal of Applied Physics, volume 29, number 2, February 1958.

[7] M. A. Levine, k. E. Marrs, J. N. Bardsley, P. Beiersdorfer, C. L. Bennett, M. H. Chen, T. Cowan,

D. Dietrich, J. R. Henderson, D. A. Knapp, A. Osterheld, B. M. Penetrante, M. B. Schneider, and J. H. Scofield.

The use of an electron beam trap in the study of highly charged ions.

Nuclear Instruments and Methods in Physics Research, volume B43, pages 431-440, September 1989.

[8] Morton A. Levine, R. E. Marrs, Robert W. Schmieder.

Measurement of instabilities and ion heating in an electrom beam ion source.

Nuclear Instruments and Methods in Physics Research, volume A237, pages 429-440, 1985.

[9] C. Litwin, M. C. Vella, and A. Sessler.

Linear electrostatic instability of the electron beam ion source.

Nuclear Instrumenis and Met'ods in Physics Research, volume 198, pages 189-192, 1982.

[10] B. M. Penetrante, M. A. Levine, J. N. Bardsley.

Computer predictions of "evaporative" cooling of highly charged ions in EBIT.

Proceedings of the Intermational Symposium on Electron Beam Ion Sources and their Applications - Upton, NY 19\$8, Amcrican Institute of Physics, Proceedings number 188.

[11] J. R. Pierce.

Theory and Design of Eiectron Beams.

D. Van Nostrand Company, 1954.

[12] Marilyn B. Schneider, Morton A. Levine, Charles L. Bennett, J. R. Henderson, D. A. Knapp and R. E. Marrs.

Evaporative cooling of highly charged ions in EBIT: an experimental realization.

Proceedings of the International Symposium on Electron Beam Ion Sources and their Applications -

Upton, NY 1988, American Institute of Physics, Proceedings No. 188.

[13] Saul Dushman.

Scientific Foundations of Vacuum Technique, pages 93-95.

John Wiley \& Sons, 2nd edition, 1962. 


\section{APPENDIX - Gas Injection System}

The gas injection system is designed to direct a sufficient number of molecules into the trapped ion region, while minimizing the number of stray molecules that enter the vacuum vessel. In order to minimize this additional gas load the system employs a differential pumping scheme.

Since their mean free path is much longer than $\ell_{1}+\ell_{2}$, gas molecules reach the outlet orifice without undergoing a collision, if their initial velocity is directed toward the aperture. Only a small fraction, the ballistic molecules, have the correct initial velocity, however. The rest strike the walls of the injection chamber, and most are carried away by the two pumps; the non-ballistic molecules that do escape through the outlet orifice can degrade the ultra-high vacuum inside EBIT. The ballistic molecules themselves do not all reach the electron beam. Their radial extent corresponds to the outlet aperture (several millimeters), while the beam is only 60 microns wide, so only a fraction intersects the beam.

Figure 9 represents the injection system. Given the aperture dimensions, pumping speeds, and the distances between the inlet and outlet apertures, one can compute both the injection rate and the leakage rate (non-ballistic molecules) into the vacuum vessel. Outgassing from the chamber walls could also affect the pressure, but appears to be negligible for the EBIT gas injector.

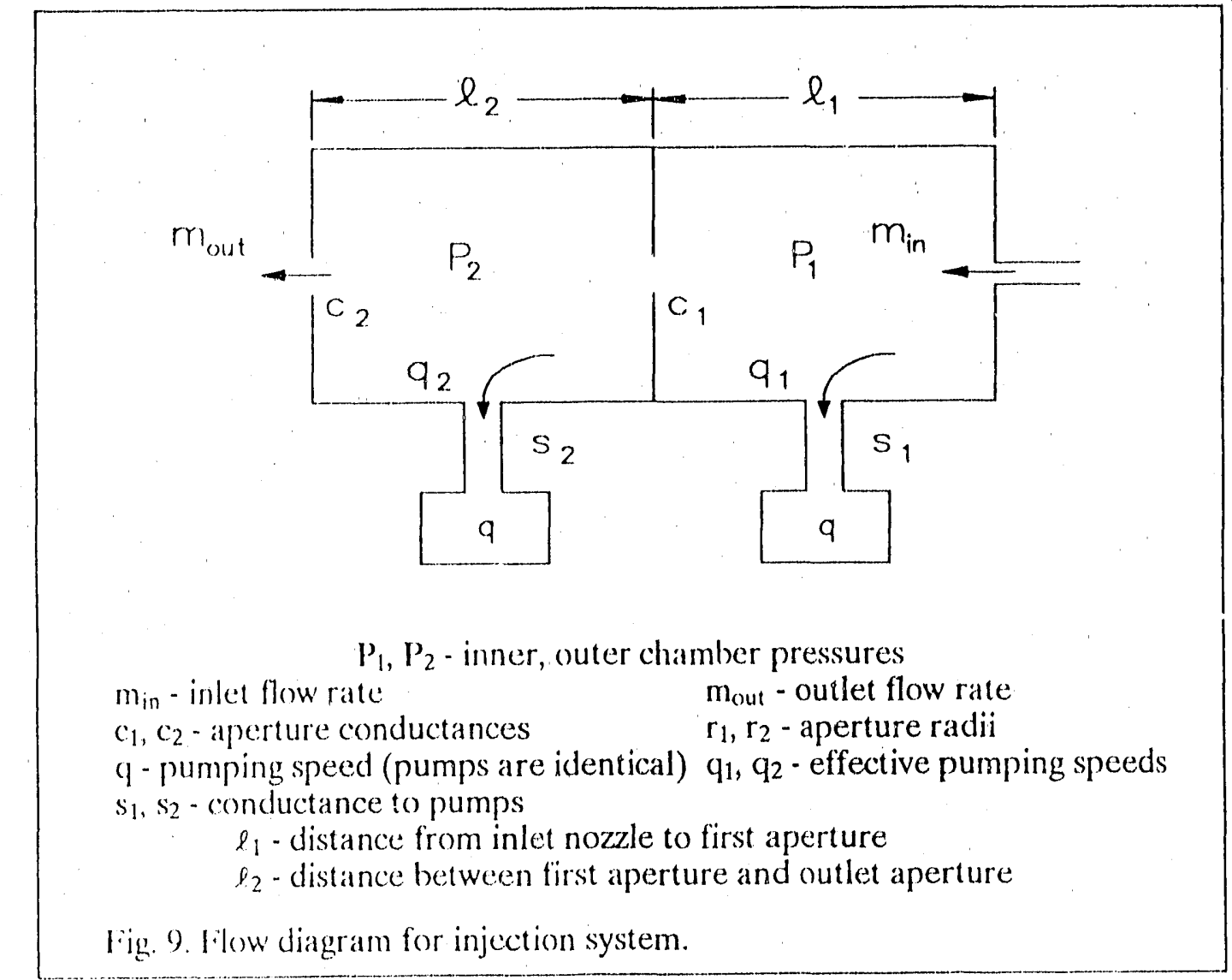

The conductances can be calculated using the formula [13]:

$$
\mathrm{s}=3638 \cdot \mathrm{kA}(\mathrm{T} / \mathrm{m})^{\frac{1}{2}}
$$


where $\mathrm{s}$ is the conductance in milliliters/second, $\mathrm{A}$ is the orifice area in $\mathrm{cm}^{2}$, T the gas temperature in Kelvin, and $m$ the gas atomic mass. $K$ is Clausing's factor, a nonlinicai function of $r / \ell$, the orifice radius divided by its length. $K=1$ for $\ell=0$, and behaves like $(8 / 3) \cdot r / \ell$ for $\ell \gg r$. The quantity $m_{\text {out }}$, which represents the non-ballistic molecules entering EBIT, is simply:

$$
\mathrm{m}_{\text {out }}=\mathrm{c}_{2} \mathrm{P}_{2}
$$

The effective pumping speeds are determined by the equations:

$$
\frac{1}{q_{1}}=\frac{1}{q}+\frac{1}{s_{1}} \quad \text { and } \quad \frac{1}{q_{2}}=\frac{1}{q}+\frac{1}{s_{2}}
$$

We can then write the steady-state mass balance equation for each chamber; thus:

$$
\begin{aligned}
m_{1 n} & =q_{1} p_{1}+c_{1}\left(p_{1}-p_{2}\right) \\
c_{1}\left(P_{1}-P_{2}\right) & =q_{2} P_{2}+c_{2} p_{2}
\end{aligned}
$$

for chambers one and two respectively. One can then solve (4) and (5) to get $P_{1}$ and $m_{1 n}$ in terms of $\mathrm{P}_{2}$. The result is:

$$
\begin{aligned}
& p_{1}=\frac{p_{2}\left(q_{2}+c_{1}+c_{2}\right)}{c_{1}} \\
& m_{1 n}=p_{2}\left[\frac{q_{1}\left(q_{2}+c_{1}+c_{2}\right)}{c_{1}}+\left(q_{2}+c_{2}\right)\right]
\end{aligned}
$$

We can also compute $m_{\text {ball }}$, the ballistic flow rate. The inlet tube emits gas molecules into a $2 \pi$ solid angle. The fraction of ballistic molecules entering EBI'T is simply the area of the exit aperture divided by the surface area of the half sphere at that distanc , i.e.:

$$
\mathrm{m}_{\text {ball }}=\mathrm{m}_{\mathrm{in}}\left[\frac{\pi \mathrm{r}_{2}^{2}}{2 \pi\left(\ell_{1}+\ell_{2}\right)^{2}}\right]
$$

If $r_{1}$ is ton small, however, the first orifice is the limiting aperture, and:

$$
m_{\text {ball }}=\ldots, m_{1 n}\left[\frac{r_{1}^{2}}{2 l_{1}^{2}}\right]
$$

Combining (2), (7), and (8), we get $m_{\text {ball }}$ in terms of $m_{\text {out }}$ :

$$
m_{\text {ball }}=m_{\text {our }}\left[\frac{r_{2}^{2}}{2\left(\ell_{1}+\ell_{2}\right)^{2}}\right] \cdot\left[\frac{q_{1}\left(q_{2}+c_{1}+c_{2}\right)}{c_{1} c_{2}}+\frac{\left(q_{2}+c_{2}\right)}{c_{2}}\right]
$$

The ratio $\mathrm{mball}_{\mathrm{b}} / \mathrm{m}_{\text {out }}$ can be considered a figure of merit for the injection system. It depends only on the geometry and pumping speeds, not on the pressure in either chamber. The maximum injection rate $m_{\text {ball }}$ will depend therefore on the inaximum additional gas load that the vacuum system can handle. Both $m_{\text {ball }}$ and $m_{\text {out }}$ are proportional $t_{0} P_{2}$. In order to maximize $m_{\text {ball }} / m_{\text {out }}, r_{1}$ and $r_{2}$ should be chosen to make expressions (8) and (9) equivalent.

In EBIT, the aperture radii are $r_{1}=1 / 16$ " and $r_{2}=3 / 32$ ", and the effective pumping speeds (for $N_{2}$ ) are $q_{1}=23$ liter $/ \mathrm{s}$ and $q_{1}=10$ liter $/ \mathrm{s}$. These give a pressure ratio $P_{1} / P_{2}=81$ and injection efficiency $m_{\text {ball }} / m_{\text {out }}=0.18$. When $P_{1}$ is set equal to $1\left(0^{-4}\right.$ Tort, $m_{\text {ball }}=1.4 \times 10^{-7}$ torr $\cdot$ liter $/ s$, or $5 \times 10^{12}$ nitrogen molecules per second. 

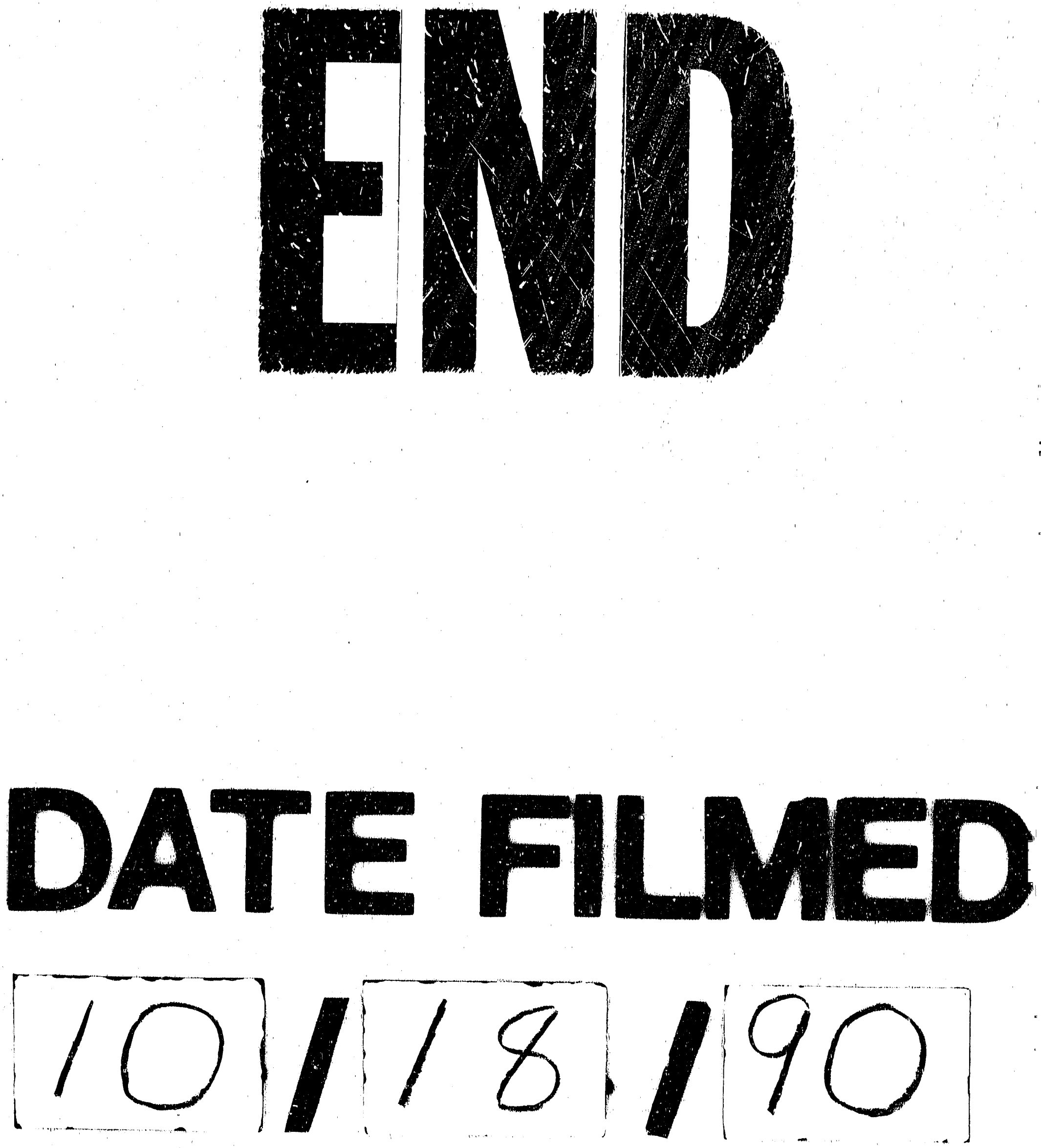
